\title{
Set-Valued Control Approach Applied to a COVID-19 Model with Screening and Saturated Treatment Function
}

\author{
Mohamed Elhia $\left(\mathbb{D},{ }^{1}\right.$ Lahoucine Boujallal $(D),{ }^{2}$ Meryem Alkama, ${ }^{3}$ \\ Omar Balatif, ${ }^{4}$ and Mostafa Rachik ${ }^{5}$ \\ ${ }^{1}$ MAEGE Laboratory, FSJES Ain Sebaa, Hassan II University, Casablanca, Morocco \\ ${ }^{2}$ MACS Laboratory, FS Ain Chock, Hassan II University, Casablanca, Morocco \\ ${ }^{3}$ BIGOF Laboratory, FSJES Ain Chock, Hassan II University, Casablanca, Morocco \\ ${ }^{4}$ Dynamical Systems Laboratory, Mathematical Engineering Team, Chouaib Doukkali University, El Jadida, Morocco \\ ${ }^{5}$ LAMS Laboratory, FS Ben M'Sik, Hassan II University, Casablanca, Morocco
}

Correspondence should be addressed to Mohamed Elhia; mohamed.elhia@gmail.com and Lahoucine Boujallal; boujallal@gmail.com

Received 11 July 2020; Revised 6 November 2020; Accepted 28 November 2020; Published 30 December 2020

Academic Editor: Chongyang Liu

Copyright (c) 2020 Mohamed Elhia et al. This is an open access article distributed under the Creative Commons Attribution License, which permits unrestricted use, distribution, and reproduction in any medium, provided the original work is properly cited.

\begin{abstract}
The purpose of this paper is modelling and controlling the spread of COVID-19 disease in Morocco. A nonlinear mathematical model with two subclasses of infectious individuals is proposed. The population is divided into five classes, namely, susceptible $(S)$, exposed $(E)$, undiagnosed infectious $\left(I_{n c}\right)$, diagnosed patients $\left(I_{c}\right)$, and removed individuals. To reflect the real dynamic of the COVID-19 transmission in Morocco, the real reported data are used for estimating model parameters. Two controls representing screening effort and limited treatment are considered. Based on viability theory and set-valued analysis, a Lyapunov function is constructed such that both exposed and infected populations are decreased to zero asymptotically. The corresponding controls are derived via a continuous selection of adequately designed feedback map. Numerical simulations are presented with three scenarios (cases when each control is used alone and the case when two controls are combined). Our results show that when only one control is to be applied, screening is the most effective in decreasing the number of people in the three infected compartments, whereas combining both controls is found to be highly effective and leads to a significant improvement in the epidemiological situation of Morocco. To the best of our knowledge, this work is the first one that applies the set-valued approach to a controlled COVID-19 model which agrees with the observed cases in Morocco.
\end{abstract}

\section{Introduction}

Coronavirus disease 2019, known as COVID-19, is a disease caused by a novel betacoronavirus named SARS-CoV-2 that affects the lower respiratory tract [1]. It is a fatal disease for patients who developed various complications including organ failure, septic shock, and pulmonary oedema (see $[1,2])$. Since its appearance in December 2019 in Wuhan, Hubei Province, China, the virus has extended internationally [3], and the number of reported cases has accelerated around the world. This is why, on 20th January 2020, the World Health Organization (WHO) declared
COVID-19 as a public health emergency of international concern [4].

Given the extent of the COVID-19 disease and the urgency to deal with it, mathematical models are a powerful tool for the management and control of this pandemic. Nowadays, the contribution of the epidemiological models in the fight against infectious diseases is indisputable. Indeed, they provide important information on the dynamics of disease transmission, give an overview of the characteristics of an epidemic, and allow predicting its future evolution and evaluating different intervention strategies to find the best control program. For more details on the use of 
mathematical models in epidemiology, we refer the interested reader to [5-10].

Several mathematical modelling studies have been proposed, which aim to understand the dynamics of this disease and provide appropriate responses to the challenges that it presents. For instance, in [11], a mathematical model considering susceptible, exposed, infected, asymptotic, quarantine/isolation, and recovered classes as in the case of COVID-19 disease was developed. Elasticity and sensitivity analyses are performed, and the global stability for the proposed model is studied. The study in [12] proposed a fractional model to describe the dynamics of COVID-19 and parametrized the model using the available infection cases. In [13], the authors proposed a compartmental model taking hospitalized and asymptomatic cases as extra compartments for COVID-19 disease. The local stability of the disease-free equilibrium in terms of the basic reproduction number is investigated, and the sensitivity analysis of the model with respect to the variation of each one of its parameters is provided. Using a generalized SEIR model and based on the public data, Peng et al. [14] estimated the key epidemic parameters and made predictions on the inflexion point and possible ending time for 5 different regions in China. Based on the collected epidemic data in China, the authors proposed in [15] an extension of the classical SEIR model. They divided the population into 6 different categories and established the time series models based on different mathematical formulas according to the variation law of the original data. Extensions of the classical SIR model by adding time delays were also proposed in $[16,17]$.

To contain the spread of COVID-19, almost all the countries affected by this pandemic have implemented severe control measures such as complete lockdown, social distancing, isolation, early detection, and the implementation of a robust system to trace contacts. In this context, some authors proposed optimal control frameworks for determining the optimal strategies that can mitigate the negative impact of this disease. The study in [18] considered a mathematical model including a quarantine class and governmental intervention measures such as lockdown, media coverage on social distancing, and improvement of public hygiene. To reduce the infected individuals as well as to minimize the cost of implementing government control measures, an optimal control problem is formulated and solved, where the control considered represents the awareness due to media coverage. The authors in [19] considered two control SEIR-type models describing the spread of COVID-19 in a human population. They used one control representing the quarantine intensity and tried to find the optimal solution by applying Pontryagin's maximum principle. In [20], a model with five compartments was proposed to highlight the role of infected people without symptoms, infected individuals with mild symptoms, and the complicated cases. Then, an optimal control problem was formulated to lessen the infected individuals by carrying out awareness campaigns, diagnosis, surveillance of airports, and quarantine of infected. In [21], the authors proposed stochastic and deterministic models to investigate the transmission mechanism of 2019-nCoV from 15 January to 5 February 2020 in Hubei province. Stochastic and deterministic analyses were performed, and optimal strategies were provided for the deterministic model. For more examples of works that address the problem of COVID19 control, we refer to [22-28].

According to Rismanbaf [29], there is still no vaccine or definitive treatment against the SARS-CoV-2 virus. However, new potential therapies are suggested based on in vitro studies, virtual screenings, and records of their effectiveness on earlier strains of coronavirus, SARS, and MERS. Furthermore, it is well known that lockdown has a colossal economic and social impact; this is why we have to think of alternative solutions. Inspired by the experience of South Korea, which has shown that mass testing is crucial to control COVID-19 disease, a solution to be explored would, therefore, be the extension of screening capacities. In this context, we propose a mathematical model that describes the COVID-19 transmission dynamics, and we investigate the impact of implementing additional treatment protocol and/ or the multiplication of the number of screening tests on the spread of this disease in Morocco. Novelty and the main contributions are as follows.

Firstly, based on the collected epidemic data and analysis of the actual situation in Morocco, we propose an extension of the classical susceptible-exposed-infectious-removed (SEIR) model. In our model, infectious people are divided into two subclasses depending on whether they are diagnosed positive and isolated or they are still undiagnosed. Considering these two categories (diagnosed and undiagnosed patients) will allow us to emphasize the importance of detection effort to fight against the COVID-19 pandemic. Furthermore, by estimating the model parameters using the real data, we obtained a more realistic model that reflects the dynamic of this disease in Morocco. Note that this model could be adapted easily to other countries.

Then, we consider two controls representing mass screening and limited treatment. For the treatment control, we use a saturated function to take into account the constraints on the health offered in this period of crisis such as insufficient human and material resources and the limitations related to the effectiveness of treatment. To investigate the impact of these intervention strategies on the spread of COVID-19, we formulate a control problem which we solve by a new direct approach. It is important to note that most of the control frameworks indicated above (paragraph 4) used an indirect approach based on the maximum principle of Pontryagin [30], which requires to design a suitable objective functional, prove the existence of an optimal solution, and solve the optimality system consisting of the state and adjoint systems that can be hard to solve. Motivated by this, we propose an alternative approach based on viability theory [31] and set-valued analysis [32]. Our approach enables the user to establish explicit formulas for the feedback controls. More precisely, we construct an appropriate Lyapunov function such that both exposed and infectious individuals decreased to zero asymptotically. The expression of our controls is obtained via a continuous selection of adequately designed feedback map.

Finally, to show the effectiveness of our theoretical results, we provide numerical simulations with different scenarios. To 
our knowledge, the present work is the first one that proposes a controlled dynamical model for COVID-19, which is fitted to the existing epidemic data in Morocco, and where the control problem is resolved using a set-valued approach.

The remainder of the paper is structured as follows: In Section 2, we introduce the compartmental model of COVID-19. Section 3 will be devoted to treat the control problem of COVID-19 by using a set-valued approach. In Section 4, we present numerical simulations with three scenarios in order to show the efficiency of our control approach. Conclusions are given in Section 5.

\section{COVID-19 Mathematical Model}

2.1. Model Construction. The model under consideration in the present paper consists of five coupled nonlinear differential equations which model the dynamic of the COVID19 disease. We consider that the total population $N$ is divided into five different compartments:

(i) Susceptible individuals ( $S$ ), people who may be infected by the virus

(ii) Exposed individuals (E), infected with the virus but without the typical symptoms of infection

(iii) Undiagnosed infectious individuals $\left(I_{n c}\right)$, who are infectious but not yet confirmed by a test

(iv) Diagnosed infectious individuals $\left(I_{c}\right)$, who are diagnosed as COVID-19-positive patients and are isolated in hospitals

(v) Removed individuals $(R)$, people who moved out either by recovery or death

We assume that the transmission of COVID-19 occurs following adequate contact between a susceptible and undetected infectious $I_{n c}$. As the infectious people in $I_{c}$ are isolated, we assume that they have no contact with the susceptible and do not transmit the disease. Due to the nonlinear contact dynamics in the population, we use the incidence function $\beta\left(I_{n c} / N\right) S$ to indicate successful transmission of COVID-19, where $\beta$ denotes the infection contact rate. We assume that all newly infected individuals enter into the exposed compartment $E$, where they reside for an average of $k^{-1}$ days ( $k$ is the rate at which individuals leave the latent class by becoming infectious). A proportion $p$ of the infectious individuals are diagnosed and isolated, and they followed a therapeutic protocol and entered the compartment $I_{c}$. The remaining infectious patients are considered as free infectious people as they are not yet detected. Among the infectious who are undiagnosed, some of them are diagnosed and isolated at a rate $\gamma$. We have observed from the clinical data that there was an increasing change within time for the number of individuals who progress from $I_{c}$ to class $R$; to reflect this, we assume that people in $I_{c}$ enter into the removed class with a time-dependent variable $r(t)$, which is derived from the following logistic function:

$$
r(t) \doteq r_{f}+\frac{r_{0}-r_{f}}{1+\exp \left(\left(t-t_{(1 / 2)}\right) / \Delta\right)},
$$

where $t_{(1 / 2)}$ represents the time at which $r(t)$ reaches its half value and $\Delta$ determines the width of $r(t)$. The parameters $r_{0}$ and $r_{f}$ model asymptotic values.
A flowchart of our model is given in Figure 1.

The dynamics of the model is governed by the following differential equations:

$$
\left\{\begin{array}{l}
\dot{S}=-\beta \frac{S I_{n c}}{N}, \\
\dot{E}=\beta \frac{S I_{n c}}{N}-k E, \\
\dot{I}_{n c}=(1-p) k E-\gamma I_{n c}, \\
\dot{I}_{c}=p k E+\gamma I_{n c}-r(t) I_{c}, \\
\dot{R}=r(t) I_{c},
\end{array}\right.
$$

with initial data

$$
\begin{gathered}
S(0)=S_{0}, \\
E(0)=E_{0}, \\
I_{n c}(0)=I_{0}^{n c}, \\
I_{c}(0)=I_{0}^{c}, \\
R(0)=R_{0} .
\end{gathered}
$$

2.2. Positivity and Boundedness of Solutions. As model (2) describes the temporal evolution of human populations, we shall show that the solutions remain nonnegative and bounded. From system (2), we have

$$
\begin{aligned}
\left.\frac{\mathrm{d} S}{\mathrm{~d} t}\right|_{S=0} & =0, \\
\left.\frac{\mathrm{d} E}{\mathrm{~d} t}\right|_{E=0} & =\beta \frac{S I_{n c}}{N} \geq 0, \\
\left.\frac{\mathrm{d} I_{n c}}{\mathrm{~d} t}\right|_{I_{\mathrm{nc}}=0} & =(1-p) k E \geq 0, \\
\left.\frac{\mathrm{d} I_{c}}{\mathrm{~d} t}\right|_{I_{c}}=0 & =p k E+\gamma I_{n c} \geq 0, \\
\left.\frac{\mathrm{d} R}{\mathrm{~d} t}\right|_{R=0} & =r I_{c} \geq 0 .
\end{aligned}
$$

We observe that all the aforementioned rates are nonnegative on bounding planes of the nonnegative cone of $\mathbb{R}^{5}$. Thus, if we begin in the interior of this cone, we shall always remain in this cone as the direction of the vector field is inward on all the bounding planes. So, all the solutions of system (2) are positive. Furthermore, as we denote the total population by $N(t), \quad N(t)=S(t)+E(t)+I_{n c}(t)+$ $I_{c}(t)+R(t)$, and by adding equations in (2), it is straightforward to prove that all solutions $S, E, I_{\mathrm{nc}}, I_{c}$, and $R$ are bounded by $N(0)$. Hence, the model is well posed in a biologically feasible domain given by the following positive invariant set: 


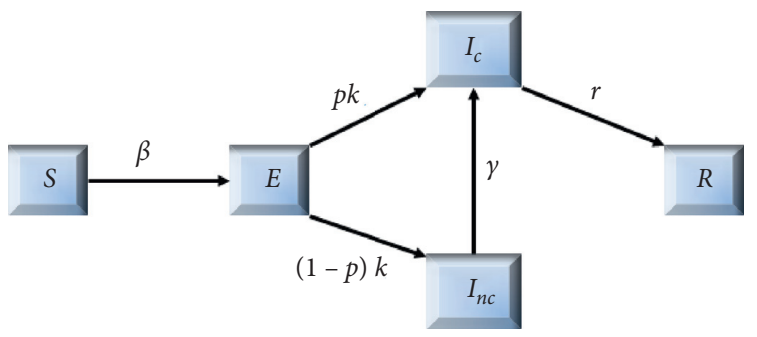

Figure 1: Transfer diagram.

$$
\mathbb{S}=\left\{\left(S, E, I_{n c}, I_{c}, R\right) \mid 0 \leq S, E, I_{n c}, I_{c}, R \leq N(0)\right\}
$$

2.3. Parameter Estimation. Let us now estimate parameters of model (2), as well as the unknown initial conditions $E_{0}$ and $I_{0}^{n c}$. Firstly, recall that the progression rate, $k$, from $E$ to the infectious stage is supposed to be equal to the inverse of the mean incubation period [10]. So, to calculate $k$, it suffices to know the average incubation period of COVID-19. The study in [33] showed that the incubation period ranges from 2 to 14 days with $95 \%$ confidence and has a mean of around 5 days. Another analysis [34] estimated the median incubation period to be 5.1 days (95\% confidence interval, 4.5 to 5.8 days). A result from [35] indicated a mean incubation period of 5.2 days (95\% CI, 4.1 to 7.0 ). Thus, we set the mean incubation period as 5 days and fix $k=1 / 5$ per day. For the remaining parameters and initial conditions $E_{0}$ and $I_{0}^{n c}$, they will be estimated by fitting the model with the collected data, communicated by the Ministry of Public Health in Morocco (see Table 1), using the MATLAB routine fminsearch. The resulting estimations are listed in Table 2, and the best model fit to the real data is shown in Figure 2. Note that, for the parameters of the function $r(t)$, the best fit to real data gives $r_{f}=0.2352, r_{0}=0.0096, t_{(1 / 2)}=70.3434$, and $\Delta=10.8262$.

\section{Control by a Set-Valued Approach}

In this section, we applied a set-valued approach to the control problem of COVID-19. For more details about this approach, we refer the reader to the works in $[38,39]$, where this approach is used to deal with the problem of asymptotic null-controllability. Note that, in [39], the proposed approach is used for epidemic models with only one infectious compartment, while the main added value here is to control the spread of the disease and decrease the number of infected people in several infectious compartments to zero asymptotically.

3.1. Control Problem Statement. In Morocco, as in several countries around the world, government authorities have opted for severe confinement as a strategic measure to limit the spread of COVID-19 in the country. Of course, this strategy is effective to avoid large losses in human lives, but it can lead to serious economic and social consequences, especially if the duration of the confinement is long. Other measures such as mass screening and treatment can also be implemented to limit the damage and allow a safe return of economic activity. To study the impact of mass screening and treatment on the dynamics of COVID-19 in Morocco, we introduce two controls $u_{1}$ and $u_{2}$ into model (2), where

(i) $u_{1}$ represents all the efforts which allow the multiplication of the number of screening tests such as the widening of diagnostic sites in professional environments in commercial and industrial units, the detection of contact subjects in family clusters, and the increase of the number of laboratories authorized to carry out COVID-19 tests.

(ii) $u_{2}$ represents the treatment of people confirmed to be COVID-19 positive and who are placed in isolation in hospitals. It is true that there is not yet a specific drug or vaccine for COVID-19, but some studies have shown that protocols combining several drugs have proven their effectiveness (a review on potential treatments for COVID-19 can be found in [29]). It is often assumed that when the resources for treatment are sufficient, the term of treatment is a linear function to infected individuals. However, in the case of COVID-19, as there are constraints and limitations in terms of medical resources, drugs, hospital beds, adequate isolation spaces, and the effectiveness of treatment, it would be more appropriate to consider a saturated treatment function $\left(u_{2} I_{c} / 1+d I_{c}\right)$, where the control $u_{2}$ is the treatment rate and $d \geq 0$ is the saturation factor that measures the effect of the infected being delayed for treatment. For examples on the use of the saturated treatment function in epidemiological modelling, we refer to [40-42].

Our model with controls is given as follows:

$$
\left\{\begin{array}{l}
\dot{S}=-\beta \frac{S I_{n c}}{N}, \\
\dot{E}=\beta \frac{S I_{n c}}{N}-k E \\
\dot{I}_{n c}=(1-p) k E-\gamma\left(1+u_{1}\right) I_{n c}, \\
\dot{I}_{c}=p k E+\gamma\left(1+u_{1}\right) I_{n c}-r(t) I_{c}-\frac{u_{2}}{1+d I_{c}} I_{c} \\
\dot{R}=r(t) I_{c}+\frac{u_{2}}{1+d I_{c}} I_{c},
\end{array}\right.
$$

with initial data

$$
\begin{gathered}
S(0)=S_{0}, \\
E(0)=E_{0}, \\
I_{n c}(0)=I_{0}^{n c}, \\
I_{c}(0)=I_{0}^{c}, \\
R(0)=R_{0} .
\end{gathered}
$$

The control in system (6) is represented by $u \doteq\left(u_{1}, u_{2}\right)$ and has values in the subset 
TABLE 1: Cumulative daily reported data of confirmed and removed cases form March 20, 2020, to May 12, 2020, reported by the Ministry of Public Health in Morocco and available in [36].

\begin{tabular}{lcccccccccccc}
\hline March & 20 & 21 & 22 & 23 & 24 & 25 & 26 & 27 & 28 & 29 & 30 & 31 \\
\hline$I_{c}$ & 86 & 96 & 115 & 143 & 170 & 225 & 275 & 345 & 402 & 479 & 556 & 617 \\
$R$ & 5 & 6 & 7 & 9 & 11 & 13 & 18 & 34 & 37 & 39 & 48 & 60 \\
\hline April & 1 & 2 & 3 & 4 & 5 & 6 & 7 & 8 & 9 & 10 & 11 & 12 \\
\hline$I_{c}$ & 654 & 708 & 791 & 919 & 1021 & 1120 & 1184 & 1275 & 1374 & 1448 & 1545 & 1661 \\
$R$ & 68 & 75 & 105 & 125 & 146 & 161 & 183 & 190 & 206 & 229 & 257 & 295 \\
\hline April & 13 & 14 & 15 & 16 & 17 & 18 & 19 & 20 & 21 & 22 & 23 & 24 \\
\hline$I_{c}$ & 1763 & 1888 & 2024 & 2283 & 2564 & 2685 & 2855 & 3046 & 3209 & 3446 & 3568 & 3758 \\
$R$ & 329 & 343 & 357 & 379 & 416 & 451 & 468 & 493 & 538 & 566 & 607 & 644 \\
\hline April & 25 & 26 & 27 & 28 & 29 & 30 & & & & & \\
\hline$I_{c}$ & 3897 & 4065 & 4120 & 4252 & 4321 & 4423 & & & & & \\
$R$ & 696 & 754 & 857 & 943 & 1096 & 1154 & & & & \\
\hline May & 1 & 2 & 3 & 4 & 5 & 6 & 7 & 8 & 9 & 10 & 11 \\
\hline$I_{c}$ & 4569 & 4729 & 4903 & 5053 & 5219 & 5408 & 5548 & 5711 & 5910 & 6063 & 6281 & 6418 \\
$R$ & 1254 & 1429 & 1612 & 1832 & 2019 & 2200 & 2362 & 2510 & 2647 & 2733 & 2999 & 3179 \\
\hline
\end{tabular}

TABle 2: Parameters and initial conditions.

\begin{tabular}{|c|c|c|c|c|}
\hline Parameter & Description & Value & Unit & References \\
\hline$\beta$ & Infection rate & 0.386 & $1 /$ day & Fitted \\
\hline$k$ & Progression rate from $E$ to the infectious stage & $1 / 5$ & $1 /$ day & Fixed \\
\hline$p$ & Proportion of the infectious that become diagnosed & 0.6936 & Dimensionless & Fitted \\
\hline$\gamma$ & Progression rate from $I_{n c}$ to $I_{c}$ & 0.0597 & $1 /$ day & Fitted \\
\hline$r(t)$ & Progression rate from $I_{c}$ to $R$ & {$[0.01-0.24]$} & $1 /$ day & Fitted \\
\hline$N$ & Total population & 35952000 & Dimensionless & {$[37]$} \\
\hline$S_{0}$ & Initial number of susceptible individuals & 35951556 & Dimensionless & Calculated \\
\hline$E_{0}$ & Initial number of exposed individuals & 111 & Dimensionless & Fitted \\
\hline$I_{n c}^{0}$ & Initial number of undiagnosed infectious individuals & 247 & Dimensionless & Fitted \\
\hline$I_{c}^{0}$ & Initial number of diagnosed and isolated individuals & 86 & Dimensionless & Table 1 \\
\hline$R_{0}^{c}$ & Initial number of removed individuals & 5 & Dimensionless & Table 1 \\
\hline
\end{tabular}

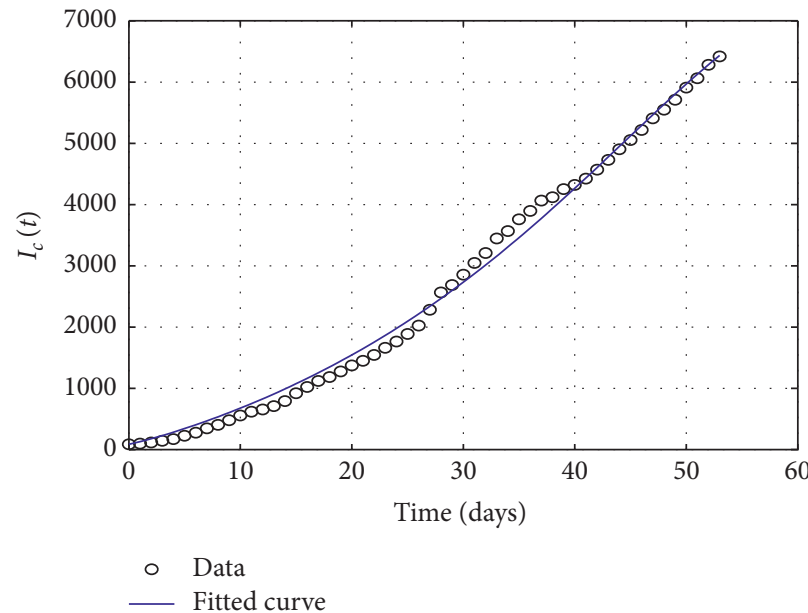

(a)

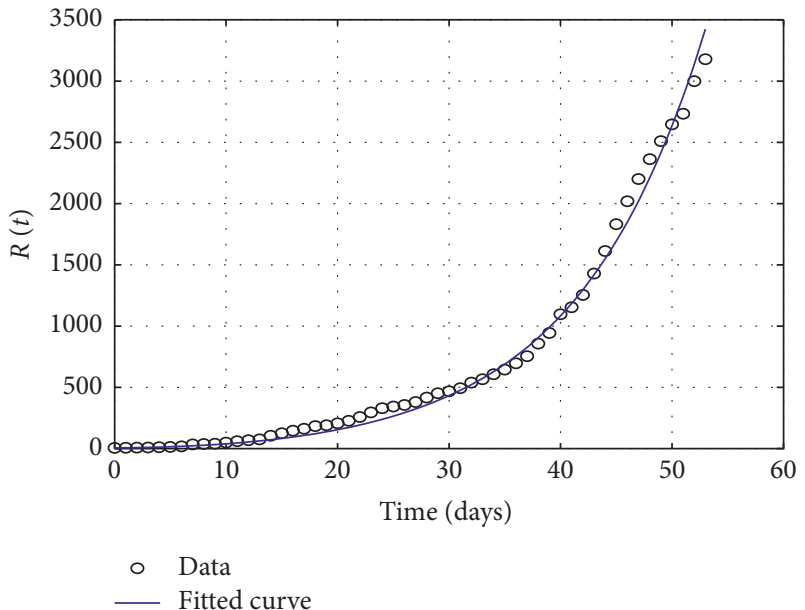

(b)

Figure 2: The reported diagnosed and removed individuals and their best fit to model (2). (a) Real data and fitted curve for $I_{c}$. (b) Real data and fitted curve for $R$. 


$$
\mathcal{U} \doteq\left[0, u_{1}^{\max }\right] \times\left[0, u_{2}^{\max }\right]
$$

where $u_{1}^{\max }$ and $u_{2}^{\max }$ are positive numbers. The aim of this paper is to reduce the number of exposed, undiagnosed, and diagnosed infectious individuals. Then, before we state our control problem, we set and transform the above nonautonomous system to the following autonomous one:

$$
\left\{\begin{array}{l}
\dot{x}=f(x, y, u), \\
\dot{y}=h(x, y, u), \\
x(0)=x_{0}, y(0)=y_{0},
\end{array}\right.
$$

where $\quad x \doteq(S, t R n, q t)^{\prime}, y \doteq\left(E, I_{n c}, I_{c}\right)^{\prime}, x_{0}=\quad\left(S_{0}, R_{0}, 0\right)$, $y_{0}=\left(E_{0}, I_{0}^{n c}, I_{0}^{c}\right)$,

$$
\left.\begin{array}{rl}
f(x, y, u) \doteq\left(\begin{array}{c}
-\beta \frac{S I_{n c}}{N} \\
r(t) I_{c}+\frac{u_{2}}{1+d I_{c}} I_{c} \\
1
\end{array}\right), \\
\beta \frac{S I_{n c}}{N}-k E \\
(1-p) k E-\gamma\left(1+u_{1}\right) I_{n c} \\
p k E+\gamma\left(1+u_{1}\right) I_{n c}-r(t) I_{c}-\frac{u_{2}}{1+d I_{c}} I_{c}
\end{array}\right) .
$$

Therefore, the control problem we have to deal with is stated as follows.

Problem: for each $\left(x_{0}, y_{0}\right) \in \Gamma \subseteq \mathbb{R}_{+}^{6}$, find a control $\bar{u}$ such that

$$
\begin{gathered}
\bar{u}:[0, \infty) \longrightarrow \mathcal{U}, \\
\lim _{s \longrightarrow \infty} \bar{y}(s)=0,
\end{gathered}
$$

where $(\bar{x}, t \bar{y})$ denotes a solution of system (9) for the control $\bar{u}$.

\section{Remark 1}

(i) It is easy to see that the solution of system (9) remains nonnegative and bounded for all $t \geq 0$; therefore, we do not need to impose (in the context of our approach) this constraint on the state of the system.

(ii) It is also noteworthy to mention that the notion of the invariant set coincides with the viability one when there exists a unique solution of system (9). We refer the interested readers to [31].

3.2. Set-Valued Approach. Now, we return to problems (11a) and (11b), which we will treat in the context of viability theory and set-valued analysis stated in Appendix. Therefore, in order to establish our control method, first we need to extend the dynamics of COVID-19 model (9) with the control dynamics governed by the following differential equation:

$$
\dot{u}=v-\alpha u,
$$

where $v$ is the new control that has values in the closed subset

$$
\mathscr{V} \doteq\left[0, \alpha_{1} u_{1}^{\max }\right] \times\left[0, \alpha_{2} u_{2}^{\max }\right]
$$

with $\alpha_{1}$ and $\alpha_{2}$ being positive numbers. If the initial state of dynamical system (12) belongs to $\mathcal{U}$, i.e., $u_{0}=u(0) \in \mathcal{U}$ and $v \in \mathscr{V}$, then the solution of differential equation (12) also belongs to $\mathcal{U}$, i.e., $\bar{u}(t) \in \mathcal{U}$ for all $t \geq 0$, since the solution of the differential equation satisfies the following inequality:

$$
u_{0}^{i} \exp \left(-\alpha_{i} t\right) \leq \bar{u}_{i}(t) \leq u_{i}^{\max }-\left(u_{i}^{\max }-u_{i}^{0}\right) \exp \left(-\alpha_{i} t\right),
$$

for $i=1,2$. According to Definition A.2, a convenient $\mathbb{R}_{+}^{3}$-Lyapunov function related to problems (11a) and (11b) can be given by

$$
\varphi(y, z)=\langle v, y\rangle+\langle\delta, z\rangle, \quad \text { for all }(y, z) \in \mathbb{R}^{3} \times \mathbb{R}^{3},
$$

where $v$ and $\delta$ denote vectors of $\mathbb{R}_{+}^{3}$ whose both coordinates are positive. Indeed, if a $\mathscr{C}^{1}$ function $w: \mathbb{R}_{+} \longrightarrow \mathbb{R}_{+}^{3}$ satisfies $\varphi(w(s), \dot{w}(s)) \leq 0$, for all $s$, then the real nonnegative function $q=\delta_{1} w_{1}+\delta_{2} w_{2}+\delta_{3} w_{3}$ is such that

$$
\dot{q}(s)+\inf _{i}\left\{\frac{v_{i}}{\delta_{i}}\right\} q(s) \leq 0, \quad \text { for all } s \geq 0 .
$$

As a result, $q(s) \longrightarrow 0$ at infinity. Thereby, both functions $w_{i}$ do so for all $i=1,2,3$.

Then, condition (11b) can be written as viability of the subset

$$
\mathscr{D}_{\varphi} \doteq\left\{(x, y, u) \in \mathbb{R}^{8}|\varphi(y, h(x, y, u))| \leq 0\right\},
$$

under the augmented control system

$$
\begin{aligned}
& \dot{x}=f(x, y, u), \\
& \dot{y}=h(x, y, u), \\
& \dot{u}=v-\alpha u .
\end{aligned}
$$

Ultimately, consider the feedback map $\mathscr{G}_{\varphi}$, for each $(x, y, u) \in \mathscr{D}_{\varphi}:$

$$
\mathscr{G}_{\varphi}(x, y, u) \dot{\doteq}\left\{v \in \mathscr{V} \mid(f(x, y, u), h(x, y, u), v-\alpha u) \in T_{\mathscr{D}_{\varphi}}(x, y, u)\right\}
$$


where $\pi_{1}$ denotes the mapping $(x, y, u) \longrightarrow(x, y)$. Then, we are ready to show the following result.
Theorem 1. Any continuous selection of the map $\mathscr{G}_{\varphi}$ provides a solution of problems (11a) and (11b) for each $\left(x_{0}, y_{0}\right) \in \Omega_{\varphi} \cap \mathbb{R}_{+}^{6}$.

Proof. For such a selection $\zeta$, it follows that

$$
\begin{array}{r}
(f(x, y, u), h(x, y, u), \zeta(x, y, u)-\alpha u) \in T_{\mathscr{D}_{\varphi}}(x, y, u), \quad \text { for all }(x, y, u) \in \mathscr{D}_{\varphi}, \\
\zeta(x, y, u) \in \mathscr{V} .
\end{array}
$$

Since $\mathscr{D}_{\varphi}$ is closed, $f, h$, and $\zeta$ are continuous; then, by using Lemma A.1, we deduce that subset $\mathscr{D}_{\varphi}$ is locally viable under system (18). Since both $f$ and $h$ have linear growth and $\zeta$ is bounded, subset $\mathscr{D}_{\varphi}$ is viable under system (18).

Now, let $\left(x_{0}, y_{0}\right)$ belong to subset $\Omega_{\varphi}$ and $u_{0}$ be such that $\left(x_{0}, y_{0}, u_{0}\right) \in \mathscr{D}_{\varphi} ;$ then, system (18) has solution $(\bar{x}, \bar{y}, \bar{u}):[0, \infty) \longrightarrow \mathscr{D}_{\varphi}, \quad$ which satisfies $(\bar{x}, t \bar{y} n, q \bar{u})(0)=\left(x_{0}, y_{0}, u_{0}\right)$.

Thanks to equation (17), this solution satisfies

$$
\varphi(\bar{y}(s), \dot{\bar{y}})(s) \leq 0, \quad \text { for all } s \geq 0 .
$$

By taking $\left(x_{0}, y_{0}\right) \in \mathbb{R}_{+}^{6}$ and using Gronwall inequality, we get $\bar{y}(s) \longrightarrow 0$ as $s \longrightarrow \infty$. Furthermore, since $\zeta(x, y, u) \in \mathscr{V}$, thanks to inequality (14), $\bar{u}(s) \in \mathscr{U}$ for all $s \geq 0$. Ending the proof, problems (11a) and (11b) have the solution from $\Omega_{\varphi} \cap \mathbb{R}_{+}^{6}$.

In what follows, we will provide the explicit expression of the feedback map $\mathscr{G}_{\varphi}(\cdot)$ of equation (19) in order to get its continuous selection as required by Theorem 1 . Now, let us set

$$
\phi(x, y, u) \doteq \varphi(y, h(x, y, u)) .
$$

Then, according to equation (15), we get

$$
\phi(x, y, u)=\langle v, y\rangle+\langle\delta, h(x, y, u)\rangle, \quad \text { for all }(x, y, u) \in \mathbb{R}^{8},
$$

and let

$$
\begin{aligned}
& \mathscr{D}_{\varphi} \dot{\dot{ }}=\left\{(x, y, u) \in \mathbb{R}^{8} \mid \phi(x, y, u) \leq 0\right\}, \\
& \nabla_{x} \phi=\left(\begin{array}{c}
\delta_{1} \beta \frac{I_{n c}}{N} \\
0 \\
-\delta_{3} r^{\prime}(t) I_{c}
\end{array}\right), \\
& \nabla_{y} \phi=\left(\begin{array}{c}
v_{1}+\left(-\delta_{1}+\delta_{2}(1-p)+\delta_{3} p\right) k \\
v_{2}+\delta_{1} \beta \frac{S}{N}+\left(1+u_{1}\right) \gamma\left(\delta_{3}-\delta_{2}\right) \\
v_{3}-\delta_{3}\left(r(t)+\frac{u_{2}}{\left(1+d I_{c}\right)^{2}}\right)
\end{array}\right), \\
& \nabla_{u} \phi=\left(\begin{array}{c}
\left(\delta_{3}-\delta_{2}\right) \gamma I_{n c} \\
-\delta_{3} \frac{I_{c}}{1+d I_{c}}
\end{array}\right) .
\end{aligned}
$$


According to Lemma A.2, we can easily get the expression of the contingent cone $T_{D_{\varphi}}(\cdot)$ as follows.
Lemma 1. For each $(x, y, u) \in \mathscr{D}_{\varphi}$, we have

$$
\begin{aligned}
& (\theta, \lambda, \Lambda) \in \mathbb{R}^{8}, \\
(\theta, \lambda, \Lambda) \in T_{D_{\varphi}}(x, y, u) \Longleftrightarrow \mid & \left\langle\nabla_{x} \phi(x, y, u), \theta\right\rangle+\left\langle\nabla_{y} \phi(x, y, u), \lambda\right\rangle \\
+ & \left\langle\nabla_{u} \phi(x, y, u), \Lambda\right\rangle \leq 0 \text { if } \phi(x, y, u)=0 .
\end{aligned}
$$

Hence, we are ready to determine a useful expression of the feedback map $\mathscr{G}_{\varphi}(\cdot)$ given by (19). To that end, we need to define the following functions and map:

$$
\begin{aligned}
& \ell_{\varphi} \doteq \nabla_{x} \phi f+\nabla_{y} \phi h-\nabla_{u} \phi \alpha u, \\
& m_{\varphi} \doteq \nabla_{u} \phi, \\
& \mathscr{C}_{\varphi}(x, y, u) \doteq\left\{v \in \mathscr{V} \mid \ell_{\varphi}(x, y, u)+\left\langle m_{\varphi}(x, y), v\right\rangle \leq 0\right\},
\end{aligned}
$$

for all $(x, y, u) \in \mathbb{R}^{8}$. It is noteworthy here that the control affine structure of the system involves that function $\ell_{\varphi}$ is affine in control $u$, and function $m_{\varphi}$ depends only from state $(x, y)$. Consequently, we are ready to state the following result.

Lemma 2. We have, for each $(x, y, u) \in \mathscr{D}_{\varphi}$,

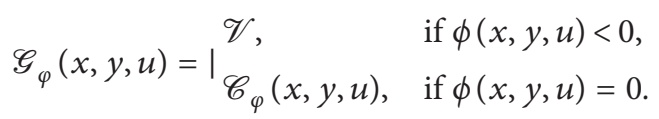

Proof. We can easily get expression (30) by using Lemma 1 and considering equations (19) and (29).
Recall that, in Theorem 1, we have proved the case where problems (11a) and (11b) have a solution from $\Omega_{\varphi} \cap \mathbb{R}_{+}^{6}$. In the next theorem, we will treat these problems from $\mathbb{R}_{+}^{6}$. Thereafter, we need to introduce the following map, for each $\sigma>0$ :

$$
\mathscr{C}_{\varphi}^{\sigma}(x, y, u) \doteq\left\{v \in \mathscr{V} \mid \ell_{\varphi}(x, y, u)+\left\langle m_{\varphi}(x, y), v\right\rangle \leq-\sigma\right\},
$$

for each $(x, y, u) \in \mathbb{R}^{8}$.

Theorem 2. Assume that, for some $\sigma>0$, the map $\mathscr{C}_{\varphi}^{\sigma}$ given by (31) has a continuous selection; then, problems (11a) and (11b) have a solution for each $\left(x_{0}, y_{0}\right) \in \mathbb{R}_{+}^{6}$

Proof. Let $\zeta$ be such a selection of the map $\mathscr{C}_{\varphi}^{\sigma}$. For each $(x, y, u) \in \mathscr{D}_{\varphi}$, this map has values included in $\mathscr{G}_{\varphi}$. Then, the selection $\zeta$ is also a selection of $\mathscr{G}_{\varphi}$. By Theorem 1 , problems (11a) and (11b) have, therefore, a solution for each $\left(x_{0}, y_{0}\right) \in \Omega_{\varphi}$. Now, we have to show that problems (11a) and (11b) have a solution from $\mathbb{R}_{+}^{6} \backslash \Omega_{\varphi}$.

Let $\left(x_{0}, y_{0}\right)$ belong to $\mathbb{R}_{+}^{6} \backslash \Omega_{\varphi}$. Thereby, $\phi\left(x_{0}, y_{0}, u\right)>0$, for all $u$, such that $\left(x_{0}, y_{0}, u\right) \in \mathbb{R}_{+}^{8}$. Let such $u_{0}$ be given, and we readily have

$$
\begin{aligned}
\frac{\mathrm{d}}{\mathrm{d} s} \phi(\bar{x}(s), \bar{y}(s), \bar{u}(s))= & \left\langle\nabla_{x} \phi(\bar{x}(s), \bar{y}(s), \bar{u}(s)), \dot{\bar{x}}(s)\right\rangle+\left\langle\nabla_{y} \phi(\bar{x}(s), \bar{y}(s), \bar{u}(s)), \dot{\bar{y}}(s)\right\rangle \\
& +\left\langle\nabla_{u} \phi(\bar{x}(s), \bar{y}(s), \bar{u}(s)), \dot{\bar{u}}(s)\right\rangle,
\end{aligned}
$$

which yields, according to equations (27) and (28),

Hence,

$$
\begin{aligned}
\frac{\mathrm{d}}{\mathrm{d} s} \phi(\bar{x}(s), \bar{y}(s), \bar{u}(s))= & \ell_{\varphi}(\bar{x}(s), \bar{y}(s), \bar{u}(s)) \\
& +\langle m(\bar{x}(s), \bar{y}(s)), \dot{\bar{u}}(s)\rangle .
\end{aligned}
$$

$$
\begin{aligned}
\phi\left(\bar{x}\left(s_{1}\right), \bar{y}\left(s_{1}\right), \bar{u}\left(s_{1}\right)\right)= & \phi\left(x_{0}, y_{0}, u_{0}\right) \\
& +\int_{0}^{s_{1}}\left[\ell_{\varphi}(\bar{x}(s), \bar{y}(s), \bar{u}(s))+\langle\zeta(\bar{x}(s), \bar{y}(s), \bar{u}(s)), m(\bar{x}(s), \bar{y}(s))\rangle\right] \mathrm{d} s .
\end{aligned}
$$


Since $\zeta$ is also a continuous selection of $\mathscr{C}_{\varphi}^{\sigma}$,

$$
\phi\left(\bar{x}\left(s_{1}\right), \bar{y}\left(s_{1}\right), \bar{u}\left(s_{1}\right)\right) \leq \phi\left(x_{0}, y_{0}, u_{0}\right)-\sigma s_{1} .
$$

Thereby,

$$
\left(\bar{x}\left(s_{1}\right), \bar{y}\left(s_{1}\right), \bar{u}\left(s_{1}\right)\right) \in \mathscr{D}_{\varphi}, \quad \text { for } s_{1} \geq \frac{\phi\left(x_{0}, y_{0}, u_{0}\right)}{\sigma} .
$$

Let $x_{1} \dot{\doteq} \bar{x}\left(s_{1}\right), y_{1} \dot{\doteq} \bar{y}\left(s_{1}\right)$, and $u_{1} \dot{\doteq} \bar{u}\left(s_{1}\right)$. As a result, the use of Theorem 1 implies that the system,

$$
\begin{aligned}
\dot{z} & =f(z, p, w), \\
z\left(s_{1}\right) & =x_{1}, \\
\dot{p} & =h(z, p, w), \\
p\left(s_{1}\right) & =y_{1}, \\
\dot{w} & =\zeta(z, p, w)-\alpha w, \\
w\left(s_{1}\right) & =u_{1},
\end{aligned}
$$

has a solution $\left.(\bar{z}, \bar{p}, \bar{w}): s_{1}, \infty\right) \longrightarrow \mathbb{R}_{+}^{6} \times \mathscr{U}$, which satisfies

$$
\bar{p}(s) \longrightarrow 0, \quad \text { when } s \longrightarrow \infty \text {. }
$$

Consequently, the control given by $\bar{u}$ on $\left[0, s_{1}\right]$ and $\bar{w}$ on $\left(s_{1}, \infty\right)$ leads to the solution of problems (11a) and (11b) from $\left\{\left(x_{0}, y_{0}\right)\right\}$.

$$
\begin{aligned}
\ell_{\varphi}(x, y, u)= & -\delta_{1}\left(\beta \frac{I_{n c}}{N}\right)^{2} S-\delta_{3} r^{\prime}(t) I_{c}+\left(\nu_{1}+\left(\delta_{3} p+\delta_{2}(1-p)-\delta_{1}\right) k\right)\left(\beta \frac{S I_{n c}}{N}-k E\right) \\
& +\left(\nu_{2}+\delta_{1} \beta \frac{S}{N}+\left(1+u_{1}\right) \gamma\left(\delta_{3}-\delta_{2}\right)\right)\left((1-p) k E-\left(1+u_{1}\right) \gamma I_{n c}\right) \\
& +\left(v_{3}-\delta_{3} r(t)\right)\left(p k E+\left(1+u_{1}\right) \gamma I_{n c}-r(t) I_{c}\right)-\left(\delta_{3}-\delta_{2}\right) \gamma I_{n c} \alpha_{1} u_{1}, \\
m_{\varphi}(x, y)= & \left(\begin{array}{c}
m_{\varphi, 1} \\
0
\end{array}\right),
\end{aligned}
$$

where $m_{\varphi, 1}=\left(\delta_{3}-\delta_{2}\right) \gamma I_{n c}$. Then, a continuous selection that provides a solution of problems (11a) and (11b) is given by

$$
\zeta_{1}(x, y, u)=\min \left(\alpha_{1} u_{1}^{\max }, \max \left(0,-\frac{\ell_{\varphi}+\sigma}{m_{\varphi, 1}}\right)\right) .
$$

\section{Numerical Simulation}

In this section, we provide some numerical simulations using MATLAB to validate the theoretical results obtained in the previous section. To investigate the impact of controls $u_{1}$ and $u_{2}$ on the spread of COVID-19 in Morocco, we will consider three different control scenarios. The first one is when the control $u_{1}$ (mass screening) is used alone. The second scenario is when only treatment (control $u_{2}$ ) is applied. The last one is when controls $u_{1}$ and $u_{2}$ are coupled. For our numerical simulations, we consider parameter's values and initial conditions given in Table 2. The control period is from March 20, 2020, to May 12, $2020\left(t_{f}=53\right.$ days). It should be noted that in order to give the continuous selection of feedback map $\mathscr{C}_{\varphi}^{\sigma}(\cdot)$, for each scenario, as expressed in equation (31), we first derive expressions of the functions $\ell_{\varphi}$ and $m_{\varphi}$ given, respectively, by equations (27) and (28). In what follows, $r^{\prime}(t)$ represents the time derivative of $r(t)$, and its expression is given by

$$
r^{\prime}(t)=\frac{\left(r_{f}-r_{0}\right) \exp \left(\left(t-t_{(1 / 2)}\right) / \Delta\right)}{\Delta\left(1+\exp \left(\left(t-t_{(1 / 2)}\right) / \Delta\right)\right)^{2}} .
$$

Scenario 1. Control with mass screening.

In this case, we assume that only mass screening, $u_{1}$, is implemented, whereas the control $u_{2}$ is set to zero. The functions $\ell_{\varphi}$ and $m_{\varphi}$ are given by
In Figures 3(a)-3(c), we depict the evolution of the exposed individuals $(E)$ and infectious individuals in compartments $\left(I_{n c}\right)$ and $\left(I_{c}\right)$ over time in both cases with and without control. We observe in these figures that mass screening significantly reduces the number of the infected people. At the end of the 53-day period, the number of the infected individuals in $E, I_{n c}$, and $I_{c}$ is reduced by $82.7 \%$, 


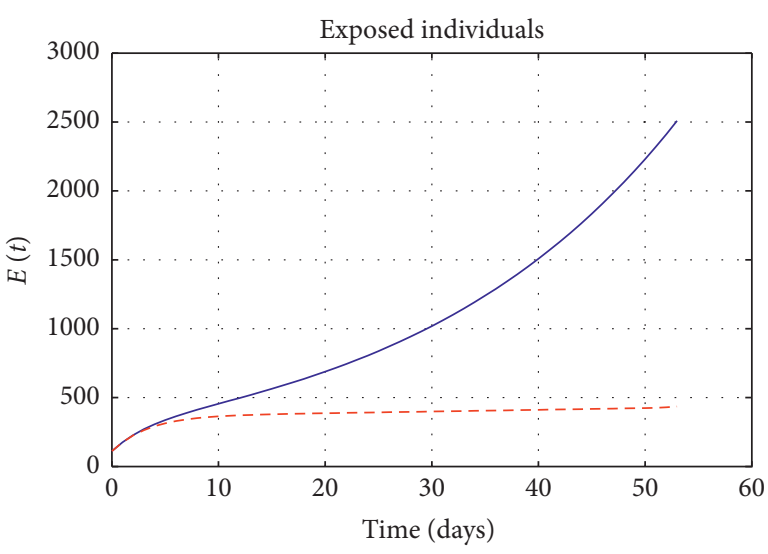

- Without control - - - With control

(a)

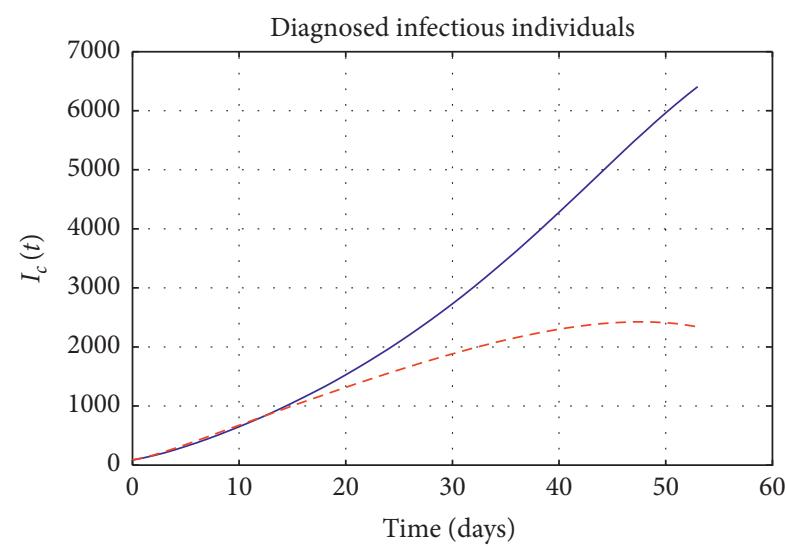

— Without control

- . - With control

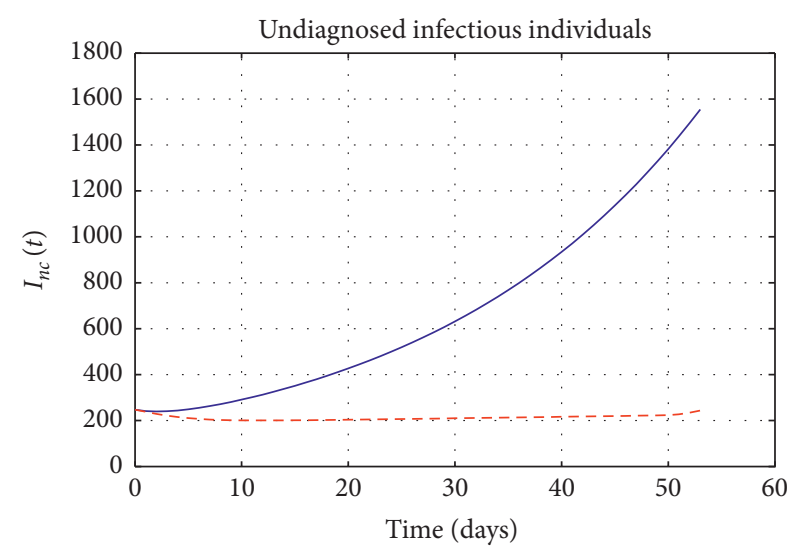

- Without control - - - With control

(b)

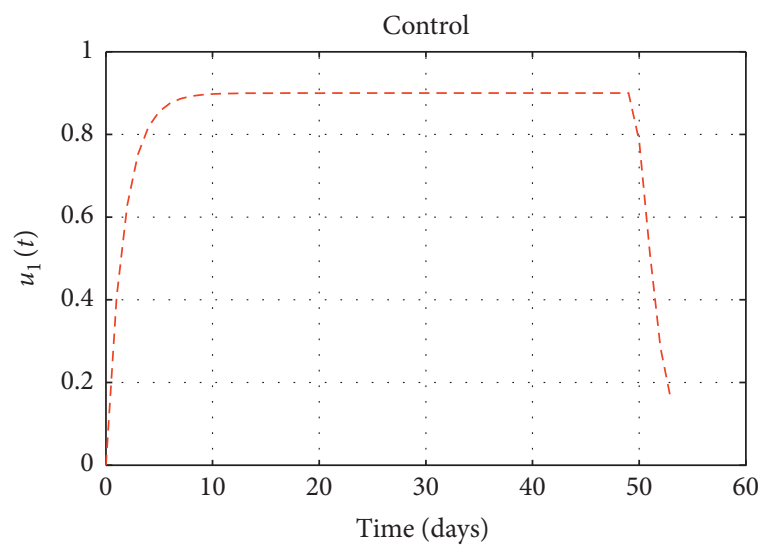

(d)

FIGURE 3: Number of $E, I_{n c}$, and $I_{c}$ with control $u_{1}$ and without control. (a) Exposed individuals $E$. (b) Undiagnosed infectious individuals $\left(I_{n c}\right)$. (c) Confirmed and isolated individuals $\left(I_{c}\right)$. (d) Evolution of the control $u_{1}$ with $\delta=v=(0.02,0.2,0.1), \alpha_{1}=0.6, \sigma=1$, and $u_{1}^{\max }=0.9$.

$84.3 \%$, and $63.4 \%$, respectively. Figure $3(\mathrm{~d})$ shows the control $u_{1}$ as a time-dependent function. We can see, in this figure, that the control $u_{1}$ should be implemented with full effort over 40 days.
Scenario 2. Control with treatment.

With this scenario, only treatment is employed as a control strategy, i.e., $u_{1}=0$. In this case, expressions of the functions $\ell_{\varphi}$ and $m_{\varphi}$ are given by

$$
\begin{aligned}
\ell_{\varphi}(x, y, u)= & -\delta_{1}\left(\beta \frac{I_{n c}}{N}\right)^{2} S-\delta_{3} r^{\prime}(t) I_{c}+\left(\nu_{1}+\left(\delta_{3} p+\delta_{2}(1-p)-\delta_{1}\right) k\right)\left(\beta \frac{S I_{n c}}{N}-k E\right) \\
& +\left(\nu_{2}+\delta_{1} \beta \frac{S}{N}+\gamma\left(\delta_{3}-\delta_{2}\right)\right)\left((1-p) k E-\gamma I_{n c}\right) \\
& +\left(v_{3}-\delta_{3}\left(r(t)+\frac{u_{2}}{\left(1+d I_{c}\right)^{2}}\right)\left(p k E+\gamma I_{n c}-r(t) I_{c}-\frac{I_{c}}{1+d I_{c}} u_{2}\right)+\delta_{3} \frac{I_{c}}{1+d I_{c}} \alpha_{2} u_{2},\right. \\
m_{\varphi}(x, y)= & \left(\begin{array}{c}
0 \\
m_{\varphi, 2}
\end{array}\right)
\end{aligned}
$$



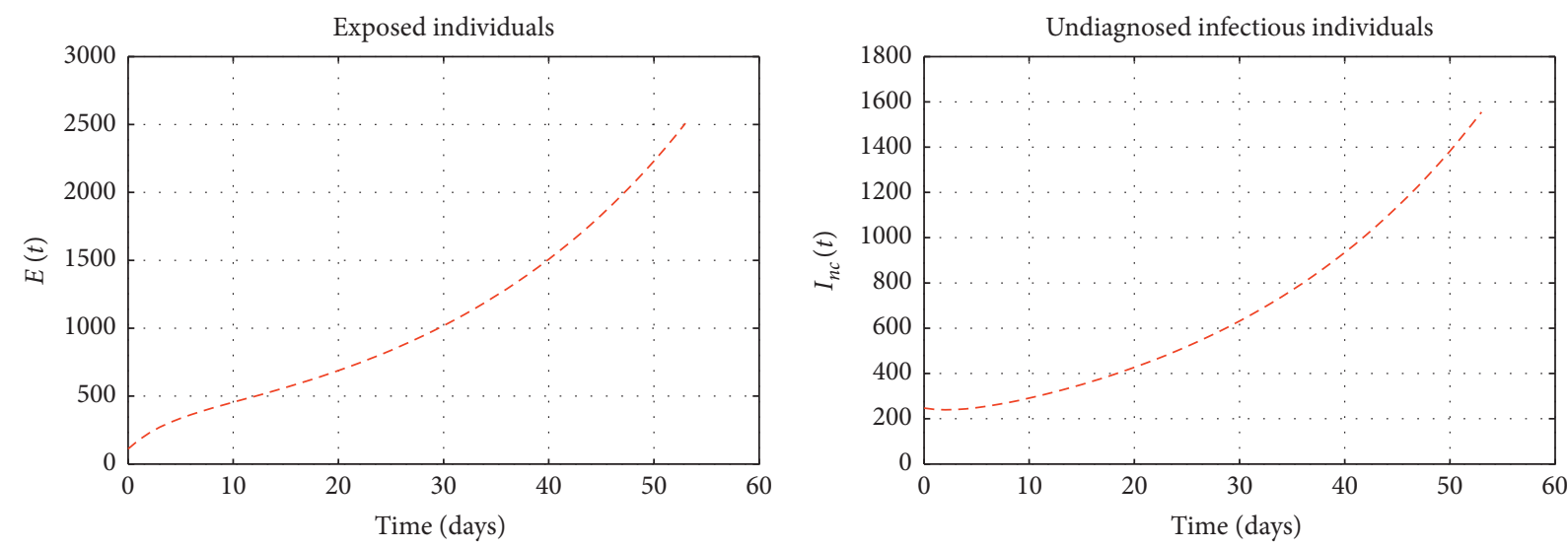

- Without contro

- .- With control

(a)

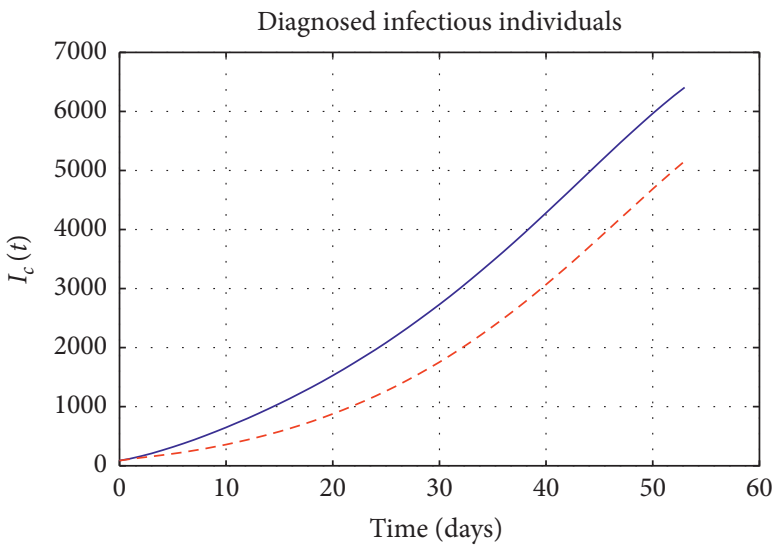

(b)

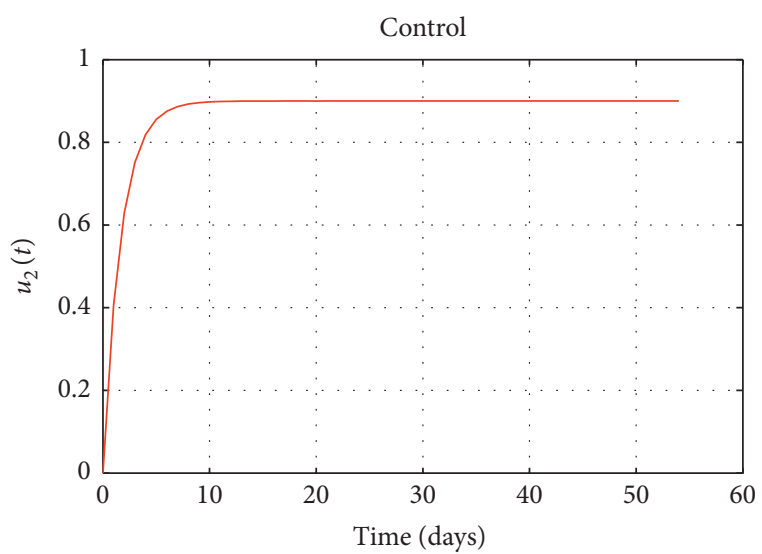

- Without control

- . - With control

(c)

(d)

Figure 4: Number of $E, I_{n c}$, and $I_{c}$ with control $u_{2}$ and without control. (a) Exposed individuals $E$. (b) Undiagnosed infectious individuals $\left(I_{n c}\right)$. (c) Confirmed and isolated individuals $\left(I_{c}\right)$. (d) Evolution of the control $u_{1}$ with $\delta=\nu=(0.02,0.2,0.1), d=0.02, \alpha_{2}=0.6, \sigma=1$, and $u_{2}^{\max }=0.9$.

where $m_{\varphi, 2}=-\delta_{3}\left(I_{c} /\left(1+d I_{c}\right)\right)$. Then, a continuous selection that provides a solution of problems (11a) and (11b) is given by

$$
\zeta_{2}(x, y, u)=\min \left(\alpha_{2} u_{2}^{\max }, \max \left(0,-\frac{\ell_{\varphi}+\sigma}{m_{\varphi, 2}}\right)\right) .
$$

Figures 4(a)-4(c) display the dynamics of the state variables $E, I_{n c}$, and $I_{c}$ with and without control $u_{2}$. It is clear from these figures that the control $u_{2}$ effectively reduces the number of people who are diagnosed and isolated, while the exposed and the undiagnosed individuals are unaffected by this control. The result in Figure 4(c) indicates that the number of people detected who are isolated decreases with treatment by $19.5 \%$ at the end of the control period. Thus, recovery of infective via treatment can reduce the burden on the health system. In addition, from Figure 4(d), one can note that, in order to reduce the number of the isolated individuals, the control $u_{2}$ should be intensively used during almost the whole control period.

Scenario 3. Control with screening and treatment.

With this approach, both controls $u_{1}$ and $u_{2}$ are used. Expressions of the functions $\ell_{\varphi}$ and $m_{\varphi}$ are given by 


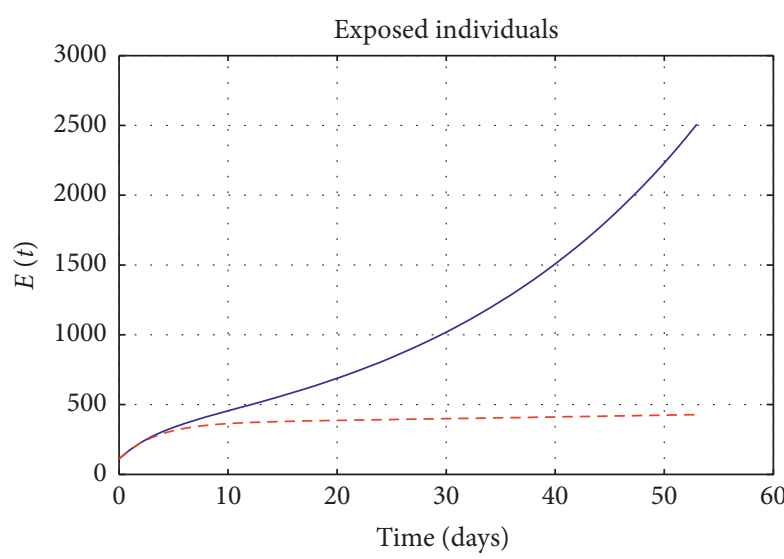

- Without control

- - - With control

(a)

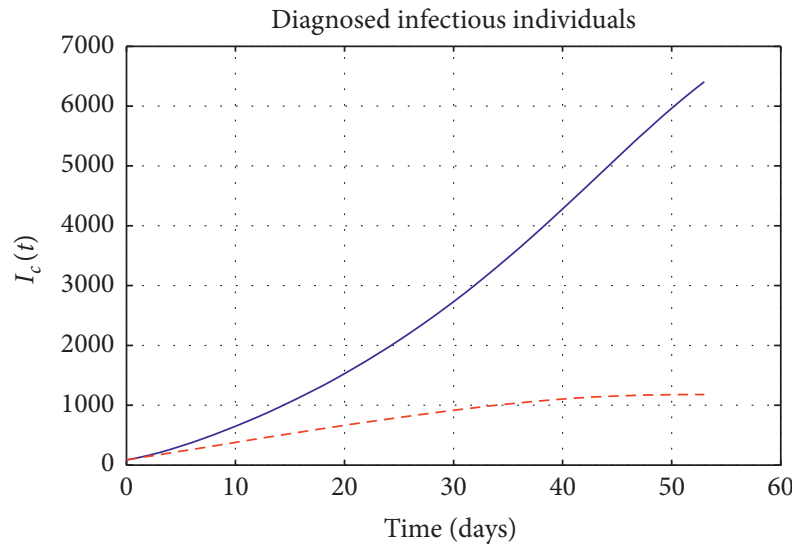

Without control

- . - With control

(c)

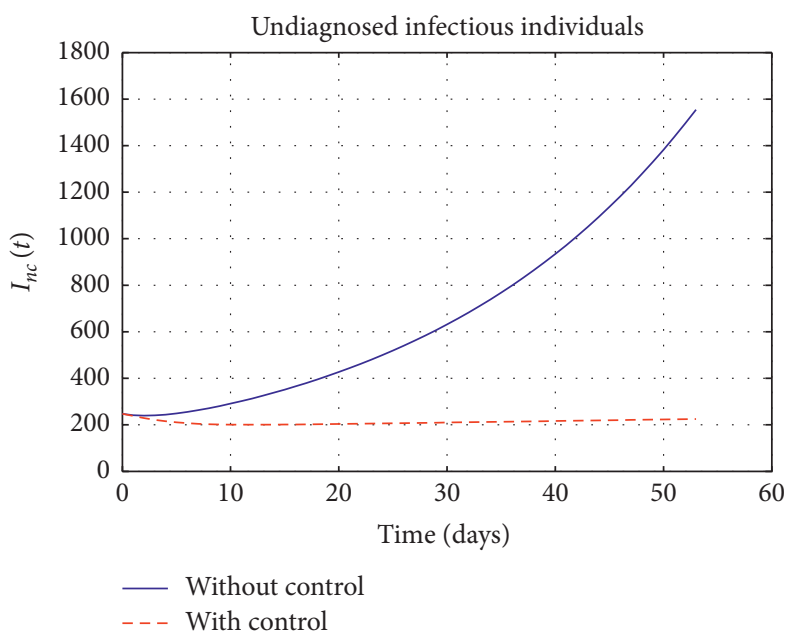

(b)

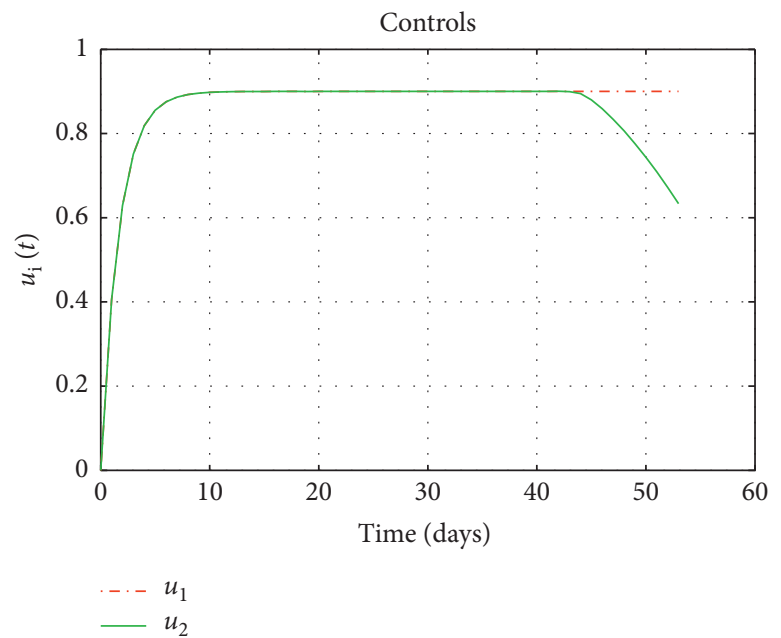

(d)

Figure 5: Number of $E, I_{n c}$, and $I_{c}$ when both controls $u_{1}$ and $u_{2}$ are applied and without control. (a) Exposed individuals $E$. (b) Undiagnosed infectious individuals $\left(I_{n c}\right)$. (c) Confirmed and isolated individuals $\left(\left(I_{c}\right)\right)$. (d) Evolution of the controls $u_{1}$ and $u_{2}$ with $\delta=v=(0.02,0.2,0.1)$, $d=0.02, \alpha=(0.6,0.6), \sigma=1$, and $u_{1}^{\max }=u_{2}^{\max }=0.9$.

$$
\begin{aligned}
\ell_{\varphi}(x, y, u)= & -\delta_{1}\left(\beta \frac{I_{n c}}{N}\right)^{2} S-\delta_{3} r^{\prime}(t) I_{c}+\left(\nu_{1}+\left(\delta_{3} p+\delta_{2}(1-p)-\delta_{1}\right) k\right)\left(\beta \frac{S I_{n c}}{N}-k E\right) \\
& +\left(\nu_{2}+\delta_{1} \beta \frac{S}{N}+\left(1+u_{1}\right) \gamma\left(\delta_{3}-\delta_{2}\right)\right)\left((1-p) k E-\left(1+u_{1}\right) \gamma I_{n c}\right) \\
& +\left(\nu_{3}-\delta_{3}\left(r(t)+\frac{u_{2}}{\left(1+d I_{c}\right)^{2}}\right)\left(p k E+\left(1+u_{1}\right) \gamma I_{n c}-r(t) I_{c}-\frac{I_{c}}{1+d I_{c}} u_{2}\right)\right. \\
& -\left(\delta_{3}-\delta_{2}\right) \gamma I_{n c} \alpha_{1} u_{1}+\delta_{3} \frac{I_{c}}{1+d I_{c}} \alpha_{2} u_{2}, \\
m_{\varphi}(x, y)= & \left(\begin{array}{c}
\left(\delta_{3}-\delta_{2}\right) \gamma I_{n c} \\
-\delta_{3} \frac{I_{c}}{1+d I_{c}}
\end{array}\right)=\left(\begin{array}{c}
m_{\varphi, 1} \\
m_{\varphi, 2}
\end{array}\right) .
\end{aligned}
$$


Then, a continuous selection that provides a solution of problems (11a) and (11b) is given by

$$
\zeta(x, y, u)=\left(\begin{array}{c}
\min \left(\alpha_{1} u_{1}^{\max }, \max \left(0,-\frac{\ell_{\varphi}+\sigma}{2 m_{\varphi, 1}}\right)\right) \\
\min \left(\alpha_{2} u_{2}^{\max }, \max \left(0,-\frac{\ell_{\varphi}+\sigma}{2 m_{\varphi, 2}}\right)\right)
\end{array}\right) .
$$

Figures 5(a)-5(c) show a comparison among the number of the infected individuals in the compartments $E, I_{n c}$, and $I_{c}$ in the uncontrolled case and when the two controls are combined. We observe, in Figures 5(a) and 5(b), that the curves are almost similar to the case when only the control $u_{1}$ is applied (Scenario 1). However, we can note from Figure 5(c) that the result obtained for the confirmed and isolated cases $\left(I_{c}\right)$ is more interesting than that in Figures 3(c) and 4(c). Thus, at the end of the 53 days, the number of people in the compartment $I_{c}$, when the controls $u_{1}$ and $u_{2}$ are used together, is reduced by $81.6 \%$ instead of $63.5 \%$ in the first scenario and $19.5 \%$ in the second scenario. Furthermore, it is important to note that when two controls are activated, the control $u_{1}$ should be used with a full effort, while the control $u_{2}$ has to be applied with its maximum level during 36 days with a gradual reduction at the end of the control period (see Figure 5(d)).

Overall, the numerical results under various interventions show that if we have to choose only one control, the screening control is the most useful because it allows a significant decrease in all the infected compartments. In addition, combining the two controls contributes positively to the improvement of the final results, particularly by reducing the number of people in the $I_{c}$ compartment.

\section{Concluding Remarks and Discussion}

In this work, we were interested in modelling and controlling the COVID-19 pandemic in Morocco. We have proposed an extension of the SEIR model that is adapted to the epidemiological data in Morocco. Thus, in addition to the susceptible $(S)$, exposed $(E)$, and removed $(R)$ compartments, we have divided infectious patients into two classes. The first class $\left(I_{c}\right)$ involves infectious people who are confirmed by a test and placed in isolation in hospitals. The second class $\left(I_{n c}\right)$ contains infectious individuals who are not yet tested. This distinction between detected and undetected people seems more realistic than considering a single compartment that includes all the infectious people. It highlights the role of screening in the dynamics of COVID19 transmission. Also, it helps to differentiate the isolated infectious who no longer contribute to the transmission of the disease from those who are still free and participate positively to the spread of COVID-19. Furthermore, to have a model that reflects as much as possible the epidemiological situation of Morocco, we have estimated the parameters of our model from official data published by the Ministry of Public Health in Morocco from March 20, 2020, to May 12, 2020.
To reduce the number of infected people in the compartments $E, I_{n c}$, and $I_{c}$, we formulate a control problem based on the proposed model to which we added two controls representing mass screening and limited treatment. This control problem is solved by a new approach based on results from the viability theory which presents the advantage of directly finding the expression of the considered controls. More precisely, we have proposed a set-valued approach based on viability theory and set-valued analysis that allows deriving controls via continuous selections of the designed feedback map.

Numerical simulations are carried out for three different scenarios, namely, the application of mass screening only, the adoption of a control strategy based on treatment only, and finally, the combination of these two intervention measures (screening and treatment). The results obtained have shown that when treatment is applied alone, it reduces only the number of people hospitalized, whereas mass screening has a significant effect on the three infected compartments $E, I_{n c}$, and $I_{c}$. Our results show that when screening is implemented alone, the number of $E+I_{n c}+I_{c}$ at the final time decreased by $71 \%$, while there is only a reduction by $19.5 \%$ when treatment is used alone. Hence, we conclude that between applying screening only and treatment only, screening is the most effective control intervention. Furthermore, combining screening and treatment is found to be better since the reduction of the total number of the infected at the final time reaches $83 \%$.

\section{Appendix}

In this section, we set some results from set-valued analysis and viability theory, in the context of nonlinear systems. Let $g: \mathbb{R}^{n} \longrightarrow \mathbb{R}^{n}$ be a given function and $D$ be a closed subset of $\mathbb{R}^{n}$. Consider the following system:

$$
\begin{aligned}
\dot{z} & =g(z), \\
z(0) & =z_{0} .
\end{aligned}
$$

Definition A.1. Subset $D$ is said to be locally viable (under system (A.1)), whenever for all $z_{0} \in D$, there exist an instant $T>0$ and a solution $\bar{z}:[0, T] \longrightarrow D$, satisfying $\bar{z}(0)=z_{0}$. If one may have $T \doteq \infty$, subset $D$ is said to be viable (under system (A.1)).

This problem can be studied in terms of contingent cones. Let $T_{D}(\cdot)$ be the contingent cone to subset $D$, given at each point $z$, as follows:

$$
T_{D}(z)=\left\{w \mid \liminf _{\varepsilon \downarrow 0} \frac{d(z+\varepsilon w, D)}{\varepsilon}=0\right\},
$$

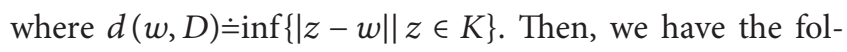
lowing results [31].

Lemma A.1. Assume that function $g$ is continuous on the closed subset $D$. Then, $D$ is locally viable under system (A.1) if and only if the following tangential condition holds: 


$$
g(z) \in T_{D}(z), \quad \text { for each } z \in D .
$$

In addition, if $g$ has a linear growth, then $D$ is viable under system (A.1).

Recall that function $g$ mapping a subset $D$ of $\mathbb{R}^{n}$ into $\mathbb{R}^{n}$ is said to be of linear growth if there exists a constant $k>0$ such that

$$
|g(z)| \leq k(1+|z|), \quad \text { for all } z \in D \text {. }
$$

Remark A.1. Nevertheless, such a condition is not required when function $g$ is bounded on subset $D$ or, in particular, when the latter subset is bounded.

Next, we present a result which was established by Aubin and Frankowska [32]. It will be fundamental for building our control laws. Let $\phi: \mathbb{R}^{n} \longrightarrow \mathbb{R}$ be a differentiable function, and consider the subset

$$
D \doteq\left\{z \in \mathbb{R}^{n} \mid \phi(z) \leq 0\right\} .
$$

Then, we have the following result.

Lemma A.2. Let $z_{0} \in D$, and suppose that the following statement holds true:

$$
\exists \xi_{0} \in \mathbb{R}^{n} \text { such that } d \phi\left(z_{0}\right) \xi_{0}<0 .
$$

Then,

$$
\begin{gathered}
\xi \in \mathbb{R}^{n}, \\
\xi \in T_{D}\left(z_{0}\right) \Leftrightarrow \mid \text { and } \\
d \phi\left(z_{0}\right) \xi \leq 0 \text { if } \phi\left(z_{0}\right)=0,
\end{gathered}
$$

where $d \phi(\cdot)$ denotes the differential operator of $\phi$.

A selection of the set-valued map $Q$ is a mapping $\zeta$ such that $\zeta(z) \in Q(z)$ for all $z$. Ultimately, the Lyapunov function considered in this paper is introduced as follows [38].

Definition A.2. Let $\mathcal{Y}$ stand for a nonempty subset of $\mathbb{R}^{N}$ and $\varphi: \mathcal{Y} \times \mathbb{R}^{N} \longrightarrow \mathbb{R}^{N}$, a $\mathscr{C}_{1}$ real-valued function. We call $\varphi$ an $\mathcal{Y}$-Lyapunov function if the following holds:

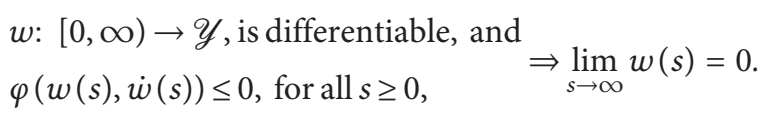

\section{Data Availability}

The data used to support the findings of this study are included within the article.

\section{Conflicts of Interest}

The authors declare that there are no conflicts of interest regarding the publication of this paper.

\section{References}

[1] C. Sohrabi, Z. Alsafi, N. O’Neill et al., "World health organization declares global emergency: a review of the 2019 novel coronavirus (covid-19)," International Journal of Surgery, vol. 76, pp. 71-76, 2020.

[2] C. Huang, Y. Wang, X. Li et al., "Clinical features of patients infected with 2019 novel coronavirus in wuhan, China," The Lancet, vol. 395, no. 10223, pp. 497-506, 2020.

[3] N. C. Peeri, N. Shrestha, M. S. Rahman et al., "The sars, mers and novel coronavirus (covid-19) epidemics, the newest and biggest global health threats: what lessons have we learned?" International Journal of Epidemiology, vol. 49, no. 3, pp. 717-726, 2020.

[4] World Health Organization, WHO Director-General's Statement on Ihr Emergency Committee on Novel Coronavirus (2019-ncov), World Health Organization, Geneva, Switzerland, 2020, https://www.who.int/dg/speeches/detail/whodirector-general-s-statement-on-ihr-emergency-committeeon-novel-coronavirus-(2019-ncov.

[5] R. M. Anderson, R. M. May, and B. Anderson, Infectious Diseases of Humans: Dynamics and Control, Vol. 28, Wiley Online Library, Hoboken, NJ, USA, 1992.

[6] H. W. Hethcote, "The mathematics of infectious diseases," SIAM Review, vol. 42, no. 4, pp. 599-653, 2000.

[7] A. Huppert and G. Katriel, "Mathematical modelling and prediction in infectious disease epidemiology," Clinical Microbiology and Infection, vol. 19, no. 11, pp. 999-1005, 2013.

[8] M. J. Keeling and P. Rohani, Modeling Infectious Diseases in Humans and Animals, Princeton University Press, Princeton, NJ, USA, 2008.

[9] F. Brauer and C. Castillo-Chavez, Mathematical Models in Population Biology and Epidemiology, Vol. 2, Springer, Berlin, Germany, 2012.

[10] M. Y. Li, An Introduction to Mathematical Modeling of Infectious Diseases, Vol. 2, Springer, Berlin, Germany, 2018.

[11] A. Mishra, S. Purohit, K. Owolabi, and Y. Sharma, "A nonlinear epidemiological model considering asymptotic and quarantine classes for sars Cov-2 virus," Chaos, Solitons \& Fractals, vol. 138, Article ID 109953, 2020.

[12] M. A. Khan and A. Atangana, "Modeling the dynamics of novel coronavirus (2019-ncov) with fractional derivative," Alexandria Engineering Journal, vol. 59, no. 4, pp. 2379-2389, 2020.

[13] F. Ndairou, I. Area, J. J. Nieto, and D. F. Torres, "Mathematical modeling of Covid-19 transmission dynamics with a case study of Wuhan," Chaos, Solitons \& Fractals, vol. 135, Article ID 109846, 2020.

[14] L. Peng, W. Yang, D. Zhang, C. Zhuge, and L. Hong, "Epidemic analysis of Covid-19 in China by dynamical modeling," 2020, https://arxiv.org/abs/2002.06563.

[15] J. Cao, X. Jiang, B. Zhao et al., "Mathematical modeling and epidemic prediction of covid-19 and its significance to epidemic prevention and control measures," Journal of Biomedical Research \& Innovation, vol. 1, no. 1, pp. 1-19, 2020.

[16] Q. Liu, D. Jiang, T. Hayat, and A. Alsaedi, "Dynamics of a stochastic sir epidemic model with distributed delay and degenerate diffusion," Journal of the Franklin Institute, vol. 356, no. 13, pp. 7347-7370, 2019.

[17] Z. Liu, P. Magal, O. Seydi, and G. Webb, "A covid-19 epidemic model with latency period," Infectious Disease Modelling, vol. 5, pp. 323-337, 2020.

[18] M. Mandal, S. Jana, S. K. Nandi, A. Khatua, S. Adak, and T. Kar, "A model based study on the dynamics of covid-19: 
prediction and control," Chaos, Solitons \& Fractals, vol. 136, Article ID 109889, 2020.

[19] E. Grigorieva, E. Khailov, and A. Korobeinikov, "Optimal quarantine strategies for covid-19 control models," 2020, https://arxiv.org/abs/2004.10614.

[20] A. Kouidere, B. Khajji, A. El Bhih, O. Balatif, and M. Rachik, "A mathematical modeling with optimal control strategy of transmission of covid-19 pandemic virus," Communications in Mathematical Biology and Neuroscience, vol. 2020, p. 24, 2020.

[21] Z. Li, Z. Teng, and C. Ma, "2019-Ncov transmission in Hubei province, China: stochastic and deterministic analyses," Complexity, vol. 2020, Article ID 9012178, 12 pages, 2020.

[22] B. Khajji, A. Labzai, A. Kouidere, O. Balatif, and M. Rachik, "A discrete mathematical modeling of the influence of alcohol treatment centers on the drinking dynamics using optimal control," Journal of Applied Mathematics, vol. 2020, Article ID 9284698, 13 pages, 2020.

[23] M. Peirlinck, K. Linka, F. S. Costabal, and E. Kuhl, "Outbreak dynamics of covid-19 in China and the United States," Biomechanics and Modeling in Mechanobiology, 2020.

[24] G. Barbosa Libotte, F. Sérgio Lobato, A. José da Silva Neto, and G. Mendes Platt, "Determination of an optimal control strategy for vaccine administration in covid-19 pandemic treatment," 2020, https://arxiv.org/abs/2004.07397.

[25] A. Perkins and G. Espana, "Optimal control of the covid-19 pandemic with non-pharmaceutical interventions," Bulletin of Mathematical Biology, vol. 82, 2020.

[26] R. Singh and R. Adhikari, "Age-structured impact of social distancing on the covid-19 epidemic in India," 2020, https:// arxiv.org/abs/2003.12055.

[27] J. Köhler, L. Schwenkel, A. Koch, J. Berberich, P. Pauli, and F. Allgöwer, "Robust and optimal predictive control of the covid-19 outbreak," 2020, https://arxiv.org/abs/2005.03580.

[28] C. Tsay, F. Lejarza, M. A. Stadtherr, and M. Baldea, "Modeling, state estimation, and optimal control for the us covid-19 outbreak," 2020, https://arxiv.org/abs/2004.06291.

[29] A. Rismanbaf, "Potential treatments for covid-19; a narrative literature review," Archives of Academic Emergency Medicine, vol. 8, no. 1, 2020.

[30] L. S. Pontryagin, Mathematical Theory of Optimal Processes, Routledge, Abingdon, UK, 2018.

[31] J.-P. Aubin, Viability Theory, Springer Science \& Business Media, Berlin, Germany, 2009.

[32] J.-P. Aubin and H. Frankowska, Set-Valued Analysis, Springer Science \& Business Media, Berlin, Germany, 2009.

[33] N. Linton, T. Kobayashi, Y. Yang et al., "Incubation period and other epidemiological characteristics of 2019 novel coronavirus infections with right truncation: a statistical analysis of publicly available case data," Journal of Clinical Medicine, vol. 9, no. 2, p. 538, 2020.

[34] S. A. Lauer, K. H. Grantz, Q. Bi et al., "The incubation period of coronavirus disease 2019 (covid-19) from publicly reported confirmed cases: estimation and application," Annals of Internal Medicine, vol. 172, no. 9, pp. 577-582, 2020.

[35] Q. Li, X. Guan, P. Wu et al., "Early transmission dynamics in Wuhan, China, of novel coronavirus-infected pneumonia," New England Journal of Medicine, vol. 382, no. 13, pp. 1199-1207, 2020.

[36] Pandémie de Covid-19 au Maroc, 2020, https://fr.wikipedia. org/wiki/Pand\%C3\%A9mie_de_Covid-19_au_Maroc.

[37] Population du Maroc Par Année Civile (en Milliers et au Milieu de l'année) Par Milieu de Résidence: 1960-2050, 2020, https://www.hcp.ma/Population-du-Maroc-par-annee-civile- en-milliers-et-au-milieu-de-l-annee-par-milieu-deresidence-1960-2050_a677.html.

[38] L. Boujallal and K. Kassara, "State-input constrained asymptotic null-controllability by a set-valued approach," IET Control Theory \& Applications, vol. 9, no. 15, pp. 2211-2221, 2015.

[39] L. Boujallal, M. Elhia, and O. Balatif, "A novel control setvalued approach with application to epidemic models," Journal of Applied Mathematics and Computing, pp. 1-25, 2020.

[40] J. Zhang, J. Jia, and X. Song, "Analysis of an seir epidemic model with saturated incidence and saturated treatment function," The Scientific World Journal, vol. 2014, Article ID 910421, 11 pages, 2014.

[41] S. Zhang, X. Meng, and X. Wang, "Application of stochastic inequalities to global analysis of a nonlinear stochastic sirs epidemic model with saturated treatment function," Advances in Difference Equations, vol. 2018, no. 1, p. 50, 2018.

[42] C.-H. Li and A. Yousef, "Bifurcation analysis of a networkbased sir epidemic model with saturated treatment function," Chaos: An Interdisciplinary Journal of Nonlinear Science, vol. 29, no. 3, Article ID 033129, 2019. 